\title{
ESQUISTOSSOMOSE MANSONI AUTÓCTONE E OUTRAS PARASITOSES INTESTINAIS EM ESCOLARES DO BAIRRO ALTO DA BOA VISTA, DA CIDADE DO RIO DE JANEIRO
}

\author{
Virginia T. Schall, Pedro Jurberg ${ }^{3}$, Henry P. F. Willcox ${ }^{2}$, Fátima G. Cavalcante ${ }^{3}$ \\ e Silvana Bagno ${ }^{3}$
}

\begin{abstract}
Procedeu-se a um levantamento coproscópico em alunos de 8 a 15 anos, pertencentes a 3 escolas do Alto da Boa Vista. De 155 alunos examinados, encontraram-se 4 casos positivos (2,6\%) de esquistossomose. Entre 25 familiares destes, encontraram-se outros 4 casos positivos (16\%). Havia na área uma população de Biomphalaria tenagophila (Orbigny, 1835), amplamente distribuida nas valas de irrigação de hortas de agrião, ligadas ao rio das Furnas. A taxa de infeç̧ão dos moluscos foi de $7(0,29 \%)$ em 2.400 examinados. Comparando-se estes resultados com dados anteriores, observa-se que a prevalência da esquistossomose se mantém no Alto da Boa Vista há pelo menos 15 anos.
\end{abstract}

Palavras chaves: Esquistossomose mansoni. Parasitos intestinais. Exame coproscópico. Inquérito malacológico.

Um estudo de Magalhães ${ }^{9}$ sobre os moluscos planorbídeos da cidade do Rio de Janeiro indica o registro de coleta de Biomphalaria sp. por vários autores em épocas e áreas diversas, embora sob denominações distintas, como, $B$. tenagophila coletada por Dunker ${ }^{4}$ no Passeio Público (sob o nome de Planorbis brasiliensis); Lutz $^{7}$ em São Cristovão e outras áreas (sob o nome de Planorbis immunis e $P$. nigricans); Pinto e Deslandes 12 em Acari e Barros Filho (sob denomição de Australorbis nigricans); Deane e cols ${ }^{3} \mathrm{em}$ Jacarepaguá ( sob a denominação de Australorbis sp.); Lucena ${ }^{6}$ em Jacarepaguá (como $A$. immunis); Andrade e Martins ${ }^{1}$ em 37 bairros ao longo das Estradas de Ferro Central do Brasil e Leopoldina (como A. immunis e $A$. tenagophilus) e Paraense e Deslandes 12 em Jacarepaguá, Manguinhos e Quinta da Boa Vista (como $A$. nigricans). A espécie $B$. glabrata também foi registrada desde 1944 restrita ao bairro de Manguinhos por vários autores e denominação diversa.

A existência de um criadouro de B. tenagophila no Alto da Boa Vista foi registrada primeiramente por

Departamento de Biologia ${ }^{1}$ e Departamento de Medicina Tropical $^{2}$ - Instituto Oswaldo Cruz - Caixa Postal 926 20 000Rio de Janeiro. Instituto de Psicologia - Laboratório de Comportamento Humano e Animal UERJ ${ }^{3}$.

Financiado pelo CNPq e FINEP

Recebido para publicação em 30/07/1984.
Magalhães ${ }^{9}$ que investigou a ocorrência de moluscos planorbídeos em todos os bairros da cidade do Rio de Janeiro. Este autor assinala a existência de exemplares infectados somente em Jacarepaguá $(B$. tenagophila - em valas de uma horta de agrião) e em um brejo em Manguinhos (B. glabrata). Anteriormente, já haviam sido encontrados caramujos infectados em Manguinhos por Paraense11, em Jacarepaguá por Deane e cols. ${ }^{3} \mathrm{e}$ em ambas as localidades por Andrade e Martins ${ }^{1}$ que detectaram focos infectados também em Barros Filho, Madureira e Oswaldo Cruz.

A ocorrência de esquistossomose no Alto da Boa Vista foi apontada por Suassuna e Coura ${ }^{14}$ que detectaram a doença em pacientes procedentes desta região, num estudo feito entre 1960 e 1968 no Rio de Janeiro. Outra evidência marcante da ocorrência desta doença na região foi demonstrada por Coura e cols ${ }^{2}$ que registraram 22 casos de esquistossomose aguda autóctone, contraida no rio das Furnas. Paes e cols ${ }^{10}$ através de um levantamento coproscópico em 1968, registraram um indice de até $13,44 \%$ de infecção por Schistosoma mansoni em escolares da regiäo e pelo inquérito malacológico, de $0,05 \%$ de caramujos positivos. Em 1969, os AA. encontraram indices de infecção ainda mais altos, sento de até $21,03 \%$ entre os escolares e $0,96 \%$ de caramujos positivos.

Recentemente, pouco se tem investigado sobre a prevalência da esquistossomose no Estado do Rio de 
Schall VT, Jurberg P, Willcox HPF, Cavalcante FG, Bagno S. Esquistossomose mansoni autóctone e outras parasitoses intestinais em escolares do bairro Alto da Boa Vista, da Cidade do Rio de Janeiro. Revista da Sociedade Brasileira de Medicina Tropical 18: 169-174, Jul-Set, 1985

Janeiro, registrando-se somente os dados da SUCAM 15 que destacam a ocorrência de 765 casos em 1981 e 693 em 1982. Entretanto, não há indicação dos bairros de procedência dos pacientes, apenas a afirmação de que no município do Rio de Janeiro, são focos comprovados da doença, o Alto da Boa Vista e Jacarepaguá.

\section{MATERIAL E MÉTODOS}

A região investigada está detalhada na Figura 1, onde se encontra a localização das hortas de agrião em cujas valas de irrigação foi encontrada a população de B. tenagophila. Estas valas se comunicam ao rio das Furnas.

Figura 1 - Planta parcial da Floresta Tijuca, com localização das Escolas e do foco de esquistossomose. Cópia da folha 286 FSecretaria Municipal de Planejamento e Coordenação Geral - Prefeitura da Cidade do Rio de Janeiro.

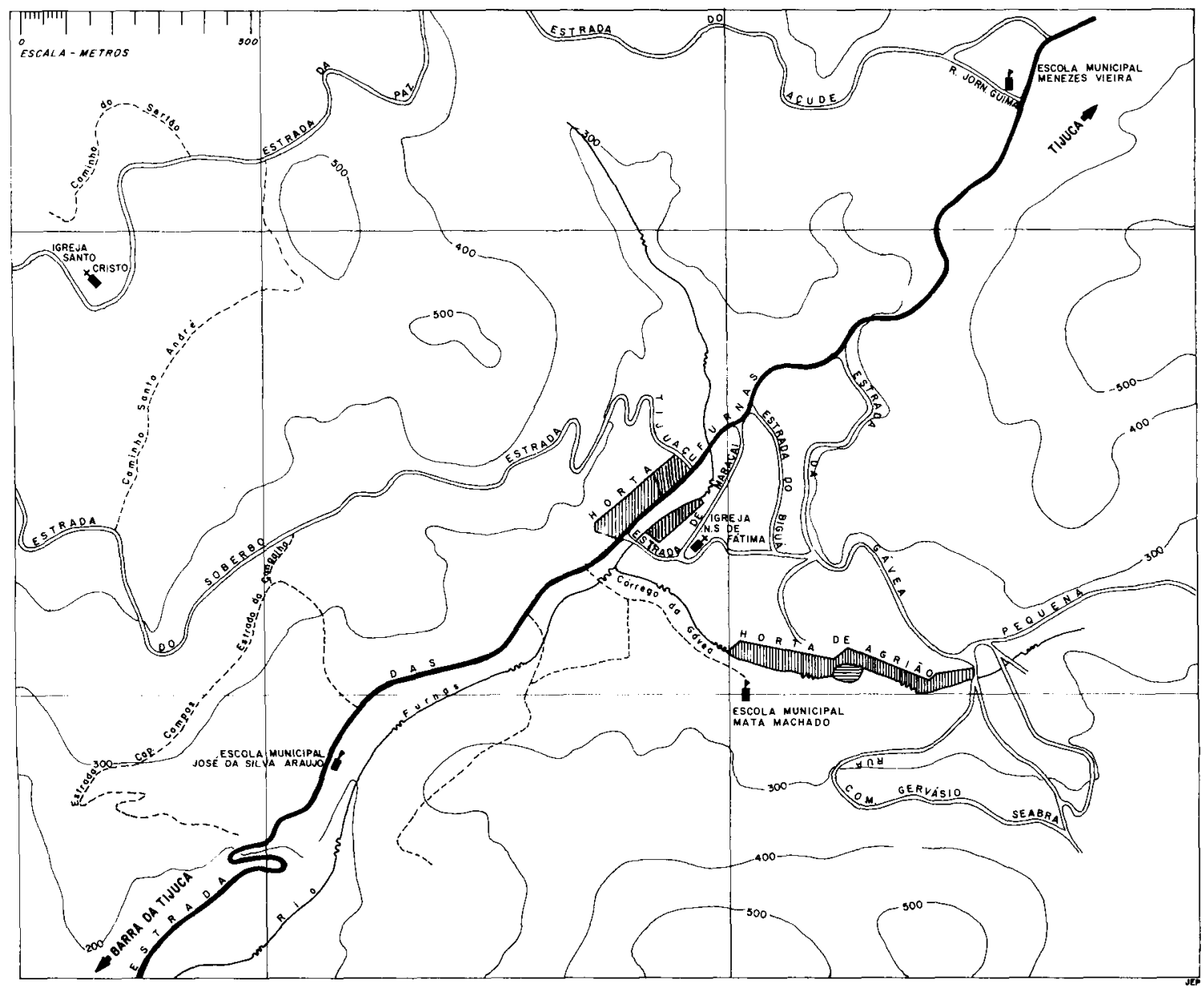

As 3 escolas investigadas estão situadas nas proximidades das hortas de agrião (Fig. 1), todas da Rede Municipal de Ensino, sendo: Escola José da Silva Araújo, Escola C.C. Mata Machado e Escola Menezes Vieira, nas quais já havia sido detectada a ocorrência de escolares com esquistossomose ${ }^{10}$. Nas duas primeiras selecionaram-se as turmas de 3 a e 4 ? séries do 1 . grau, e na terceira, as turmas de 6a série, para inquérito coproscópico, tendo sido o material examinado pelo método de sedimentação qualitativa, de Lutz ${ }^{9}$. A amostra examinada totalizou 155 alunos de 8 a 15 anos de idade.

Em paralelo, realizou-se um levantamento malacológico nas hortas de agrião adjacentes à Escola C.C. Mata Machado. Os exemplares coletados foram 
Schall VT, Jurberg P, Willcox HPF, Cavalcante FG, Bagno S. Esquistossomose mansoni autóctone e outras parasitoses intestinais em escolares do bairro Alto da Boa Vista, da Cidade do Rio de Janeiro. Revista da Sociedade Brasileira de Medicina Tropical 18: 169-174, Jul-Set, 1985

colocados sob iluminação artificial durante cerca de 3 horas para posterior exame sob lupa da possivel existência de cercárias de $S$. mansoni.

Anteriormente à coleta de fezes foram feitas entrevistas com um terço dos alunos, escolhidos por tabela de números aleatórios, para investigação de conceitos e cuidados com a saúde e hábitos de higiene. Após os resultados dos exames, as familias dos alunos portadores de esquistossomose foram procurados para investigação epidemiológica, tendo sido coletadas fezes de todos os individuos para exame.

\section{RESULTADOS}

Ocorrência da esquistossomose. O levantamento malacológico possibilitou a coleta de 2400 exemplares de $B$. tenagophila, identificação confirmada pela Dra. Lygia R. Corrêa - Departamento de Malacologia - Instituto Oswaldo Cruz, encontrandose 7 positivos para cercárias de $S$. mansoni $(0,29 \%)$.

Do total de 155 alunos examinados foram identificados 4 , do sexo masculino, eliminando ovos de $S$. mansoni, sendo 2 da Escola José da Silva Araujo (com 10 e 15 anos), 1 da Escola Mata Machado (11 anos) e 1 da Escola Menezes Vieira (12 anos). Verificou-se que 3 desses escolares residem nas áreas de Mata Machado (1) e Maracai (2), sendo que o quarto, mudou-se há dois anos desta área para a Estrada do Soberbo, também no Alto da Boa Vista.

As residências dos três primeiros foram visitadas, tendo sido encontradas condições sanitárias precárias, como poucas casas ligadas à rede d'água e ao sistema de esgotos. Do contato com as familias destes alunos, foi apurado que eles nasceram no Rio de Janeiro, de onde nunca se ausentaram, tendo residido sempre no Alto da Boa Vista.

Pelos exames coproscópicos de 25 familiares dos alunos portadores de esquistossomose, encontrouse outros 4 casos positivos (16\%), todos residentes na área de Maracai, sendo 3 do sexo feminino, com 15 , 16 e 34 anos, e 1 do sexo masculino, com 13 anos. Pelo Método de Kato adaptado por Katz e cols ${ }^{5}$ encontrou-se o índice de 72 e 384 ovos/g de fezes de 2 examinados (sexo feminino, com 15 e 16 anos respectivamente).

Um breve levantamento epidemiológico revelou que os alunos positivos têm freqüente contato com águas do córrego da Gávea Pequena ou do rio das

Tabela 1 - Prevalência de parasitos intestinais em escolares de 8-15 anos de um bairro do Rio de Janeiro (Alto da Boa Vista).

Escolas José da S. Araujo Mata Machado Menezes Vieira Total

(n.o examinados: 78) (n.o examinados: 27) (n.o examinados: 27) (n.oxaminados: 155)

\begin{tabular}{lrrrrrrrr}
\hline & \multicolumn{2}{c}{ Positivos } & \multicolumn{2}{c}{ Positivos } & \multicolumn{2}{c}{ Positivos } & \multicolumn{2}{c}{ Positivos } \\
\cline { 2 - 9 } Intestinais & \multicolumn{1}{c}{$N}$. & \multicolumn{1}{c}{$\%$} & \multicolumn{1}{c}{$N^{o}$} & \multicolumn{1}{c}{$\%$} & $N^{o}$ & $\%$ & $N$. & $\%$ \\
\hline Ancilostomídeos & 9 & 11,5 & 2 & 4,0 & 1 & 3,7 & 12 & 7,7 \\
A. lumbricoides & 46 & 58,9 & 23 & 46,0 & 6 & 22,2 & 75 & 48,4 \\
E. vermiculares & 2 & 2,6 & 1 & 2,0 & - & - & 3 & 1,9 \\
H. nana & - & - & 1 & 2,0 & - & - & 1 & 0,6 \\
S. mansoni & 2 & 2,6 & 1 & 2,0 & 1 & 3,7 & 4 & 2,6 \\
S. stercoralis & 5 & 6,4 & 2 & 4,0 & - & - & 7 & 4,5 \\
T. trichiura & 27 & 34,6 & 23 & 46,0 & 9 & 33,3 & 59 & 38,0 \\
E. coli & 15 & 19,2 & 5 & 10,0 & 2 & 7,4 & 22 & 14,2 \\
E. histolytica & 3 & 3,8 & 4 & 8,0 & 2 & 7,4 & 9 & 5,8 \\
E. nana & 22 & 28,2 & 10 & 20,0 & 5 & 18,5 & 37 & 23,9 \\
G. lamblia & 11 & 14,1 & 6 & 12,0 & 2 & 7,4 & 19 & 12,3 \\
I. butschilli & 2 & 2,6 & 1 & 2,0 & - & - & 3 & 1,9 \\
\hline \multicolumn{1}{c}{ Total } & 144 & $\ldots$ & 79 & $\ldots$ & 28 & $\ldots$ & 251 & $\ldots$ \\
\hline
\end{tabular}


Schall VT, Jurberg P, Willcox HPF, Cavalcante FG, Bagno S. Esquistossomose mansoni autóctone e outras parasitoses intestinais em escolares do bairro Alto da Boa Vista, da Cidade do Rio de Janeiro. Revista da Sociedade Brasileira de Medicina Tropical 18: 169-174, Jul-Set, 1985

Furnas, e as mulheres, têm por hábito lavar roupas no segundo.

Todos os casos positivos foram encaminhados ao Posto de Saúde local para tratamento, o que se realizou com o auxilio da SUCAM.

Ocorrência de outros parasitos intestinais. A prevalência de outros parasitos intestinais foi investigada para a amostra de 155 alunos e para os 25 familiares dos alunos portadores de esquistossomose
(Tabela 1). Os exames foram positivos para 12 espécies de parasitos, dentre os quais, os de maior incidência foram: Ascaris lumbricoides (48,4\%), Endolimax nana $(23,5 \%)$ e Trichuris trichiura (38\%).

A Tabela 2 apresenta os dados relativos aos 25 familiares dos alunos com esquistossomose. Das 4 espécies de parasitos encontrados, a maior incidência foi de Ascaris lumbricoides (64\%), E. coli (16\%), S. mansoni $(16 \%)$ e $T$. trichiura $(77 \%)$.

Tabela 2 - Prevalência de Parasitos Intestinais de 25 familiares de alunos portadores de esquistossomose.

\begin{tabular}{|c|c|c|c|c|c|c|}
\hline \multirow{3}{*}{$\begin{array}{c}\text { Sexo } \\
\text { Parasitos }\end{array}$} & \multirow{2}{*}{\multicolumn{2}{|c|}{$\begin{array}{c}\begin{array}{c}\text { Masculino } \\
n=12\end{array} \\
\text { Positivos }\end{array}$}} & \multirow{2}{*}{\multicolumn{2}{|c|}{$\begin{array}{c}\begin{array}{c}\text { Feminino } \\
n=13\end{array} \\
\text { Positivos }\end{array}$}} & \multirow{2}{*}{\multicolumn{2}{|c|}{$\begin{array}{c}\begin{array}{c}\text { Total } \\
n=25\end{array} \\
\text { Positivos }\end{array}$}} \\
\hline & & & & & & \\
\hline & No & $\%$ & $N o$ & $\%$ & $N o$ & $\%$ \\
\hline Ancilostomideos & 一 & - & 1 & 7,7 & 1 & 4,0 \\
\hline A. lumbricoides & 11 & 91,6 & 5 & 38,5 & 16 & 64,0 \\
\hline E. coli & 1 & 8,3 & 3 & 23,0 & 4 & 16,0 \\
\hline E. histolytica & 1 & 8,3 & - & - & 1 & 4,0 \\
\hline E. nana & 2 & 16,7 & - & - & 2 & 8,0 \\
\hline G. lamblia & - & - & 2 & 15,4 & 2 & 8,0 \\
\hline S. mansoni & 1 & 8,3 & 3 & 23,0 & 4 & 16,0 \\
\hline S. stercoralis & 1 & 8,3 & 1 & 7,7 & 2 & 8,0 \\
\hline T. trichiura & 8 & 66,7 & 10 & 76,9 & 18 & 77,0 \\
\hline Total & 25 & $\ldots$ & 25 & .. & 50 & $\ldots$ \\
\hline
\end{tabular}

Obs.: $\overline{\mathrm{X}}$ parasito/pessoa: 2,0

\section{DISCUSSÃO}

Paes e cols ${ }^{10}$, em 1970 , encontraram os seguintes indices de prevalência da esquistossomose: $5,32 \%$ na Escola José da Silva Araujo; $4,2 \%$ na E. Menezes Vieira e $21,03 \%$ na E. Mata Machado, superiores aos obtidos agora (Tabela 1 ).

Entretanto, nos 25 familiares dos alunos com esquistossomose, encontrou-se uma taxa de positividade de $16 \%$, semelhante à encontrada por Paes e cols. ${ }^{10}$, em 1970 , no levantamento feito com a população do bairro.
Permaneceram as condições favoráveis à transmissão da doença na Região. Além disso, como mostraram Coura e $\operatorname{cols}^{2}$, os caramujos podem ser carreados pelas chuvas, das hortas ao rio das Furnas, contribuindo para a disseminação da doença, principalmente em época de verão, quando muitas pessoas costumam banhar-se aí.

A alta prevalência de outros parasitos intescinais, nos escolares e nas familias investigadas, denota más condições sanitárias na região, onde também o clima, caracterizado por grande umidade e, em certas épocas, altas temperaturas, favorece a disseminação destes parasitos. Tais resultados são semelhantes aos encontrados por Paes e cols. ${ }^{10}$ 
Schall VT, Jurberg P, Willcox HPF, Cavalcante FG, Bagno S. Esquistossomose mansoni autóctone e outras parasitoses intestinais em escolares do bairro Alto da Boa Vista, da Cidade do Rio de Janeiro. Revista da Sociedade Brasileira de Medicina Tropical 18: 169-174, Jul-Set, 1985

\section{SUMMARY}

The present situation regarding schistosomiasis mansoni prevalence among primary school children was evaluated in the periphery of Rio de Janeiro city (Alto da Boa Vista). A coproscopic survey was carried out with students of 8 to 15 years old from 3 schools of the area. Among 155 examined, 4 positive cases $(2,6 \%)$ of schistosomiasis were found. On examining 25 relatives of the positive students, 4 other positives cases $(16 \%)$ were found, all from the same region. The malacological survey carried out revealed a Biomphalaria tenagophila population largely distributed in the irrigation ditches of water-cress gardens surrounding the Furnas River. Seven (7) out of 2400 snails collected were positive for $\mathrm{Schistosoma}$ mansoni $(0,29 \%)$. Comparing these results with previous data, it can be observed that schistosomiasis prevalence has been maintained in this area for as long as 15 years. Stool examinations revealed a high prevalence of $\mathrm{A}$. lumbricoides $(48,4 \%)$. E. nana $(23,5 \%)$ and $\mathrm{T}$. trichiura (38\%).

Key words: Schistosomiasis mansoni. Intestinal parasites. Coproscopic survey. Malacological survey.

\section{AGRADECIMENTOS}

Os autores agradecem ao Dr. Luis Rey, pela leitura crítica do manuscrito. Aos estagiários do Departamento de Biologia, Mauricio Carvalho de Vasconcellos, Elizabeth Maria de Almeida, Clarice Casz e Kátia S. Valente, pelo auxilio na fase de coleta de dados. Ao técnico de pesquisa, Júlio Cesar Miguel, pela coordenação dos exames coproscópicos. Às diretoras das escolas investigadas: professoras Maria Cristina Burgos Ribeiro, Maria Coeli S.M. Pontes e Luce Diegues, pela colaboração. À Dra. Lilia Machado de Moraes, responsável pela Unidade Auxiliar de Cuidados Primários de Saúde (UACPS) do Alto da Boa Vista e aos Drs. Anisio José dos Santos e Sonia Sanches, pelo atendimento médico aos escolares. A José Eduardo Prado, pelo desenho do mapa.

\section{REFERÊNCIAS BIBLIOGRÁFICAS}

1. Andrade RM, Martins RS. Contribuição para o conhecimento dos criadouros de planorbideos no Distrito Federal. II. Resultado geral das pesquisas efetuadas para a localização de focos de transmissão de esquistos- somose mansoni. Revista Brasileira de Malariologia e Doenças Tropicais 8: 379-385, 1956.

2. Coura JR, Camillo-Coura L, Kalache A, Argento CA Esquistossomose aguda autóctone de foco na cidade do Rio de Janeiro. Estudo de 22 casos. Revista da Sociedade Brasileira de Medicina Tropical, 4: 387-397, 1970.

3. Deane LM, Martins RS, Lalo MB. Um foco ativo de esquistossomose mansônica em Jacarepaguá, Distrito Federal Revista Brasileira de Malariologia e Doenças Tropicais 5: 249-252, 1953.

4. Dunker W. Die Familie der Limnaeiden, etc. In: Martini \& Chemnitz: Systematische Conchylien-Cabinet V. 17, Bauer \& Nürnberg, 1856

5. Katz N, Chaves A, Pellegrino J. A simple device for quantitative stool thick-smear technique in $S$. mansoni. Revista do Instituto de Medicina Tropical de São Paulo, 14: 397-400, 1972.

6. Lucena DT. Resenha sistemática dos planorbideos brasileiros 104 pp. Gráfica Editora Recife S.A., Recife, 1956.

7. Lutz A. Caramujos de água doce de gênero Planorbis, observados no Brasil. Memórias do Instituto Oswaldo Cruz, 10: 65-82, 1918.

8. Lutz A. O Schistosomum e a schistosomatose segundo observações feitas no Brasil. Memórias do Instituto Oswaldo Cruz 11: 121-150, 1919.

9. Magalhães LA. Moluscos planorbideos do Estado da Guanabara. Revista Brasileira de Biologia 24: 277-288, 1964.

10. Paes R, Menezes ZB, Camargo S. Um novo foco de esquistossomose na Guanabara, Alto da Boa Vista. Revista Brasileira de Malariologia e Doenças Tropicais, 22: 203-229, 1970.

11. Paraense WL. Observações adicionais sobre o sexo do Schistosoma mansoni nas infestaçōes por cercárias de um único molusco. Memórias do Instituto Oswaldo Cruz 47: 535-546, 1949.

12. Paraense WL, Deslandes N. The renal ridge as a reliable character for separating Taphius glabratus from Taphius tenagophilus. The American Journal of Tropical Medicine and Hygiene 8: 456-472, 1959.

13. Pinto DS, Deslandes N. Contribuição ao estudo da sistemática de planorbídeos brasileiros. Revista de Serviço Especial de Saúde Pública 6: 135-167, 1953. 
Schall VT, Jurberg P, Willcox HPF, Cavalcante FG, Bagno S. Esquistossomose mansoni autóctone e outras parasitoses intestinais em escolares do bairro Alto da Boa Vista, da Cidade do Rio de Janeiro. Revista da Sociedade Brasileira de Medicina Tropical 18: 169-174, Jul-Set, 1985

14. Suassuna A, Coura JR. Esquistossomose mansoni no Estado da Guanabara. Aspectos epidemiológicos relacionados às migraçōes internas. Revista da Sociedade Brasileira de Medicina Tropical 3: 59-71, 1969.
15. SUCAM. Algumas consideraçōes sobre o comportamento das endemias controladas pela SUCAM no Rio de Janeiro. Relatório Anual. Diretoria Regional do Rio de Janeiro. 3pp datilografada, 1982. 